\title{
An early Ptolemaic bank register from the Arsinoite nome revised
}

\author{
Willy Clarysse (Leuven), Dorothy J. Thompson (Cambridge) \\ and Laurent Capron (Paris)
}

\begin{abstract}
New readings and a rearrangement of the fragments have led to this reedition of an Arsinoite bank register, first published two years ago (APF 55/2 [2009] 230-250). The register covers one month in year 12 (of Ptolemy III). A wide variety of taxes is recorded, paid by both civilian and military sectors at different local banks; details are here collected in a central register most probably from the nome capital. The surviving text records the final 5-6\% of inpayments for the current year, followed by arrears going back year-by-year. Different agios are charged on different taxes. The large sums on the back are probably annual payments relating to the whole nome.
\end{abstract}

Keywords: Taxes, bank, register, money, military

In APF 55 (2009), pp. 230-260 Willy Clarysse and Dorothy Thompson published a complex list of tax inpayments made to a bank, probably in Krokodilon polis (P.Sorb. inv. 371). The text consisted of numerous fragments, which were painstakingly puzzled together during three study visits to the Sorbonne collection. However, when Laurent Capron returned from leave and started making digital photographs of the whole text he succeeded in placing one more loose fragment and also established that Fragment B of the earlier publication immediately preceded Fragment A, with the last column of Fragment B continuing in the first column of Fragment A. Gradually the other fragments could also be placed, so that four columns are now more or less complete. More fragments may still be hidden in the collection, but there was no time for a systematic search. The new joins are beyond doubt, as can be seen in the new arrangement on the Sorbonne website:

$<$ <ttp://www.papyrologie.paris4.sorbonne.fr/photos/2010371.tif>.

As a result the line numbering of the whole has to be changed. Frag. B now precedes and 11.148-209 have become 11.1-62. Lines 1-7 (old Frag. A) of the editio princeps now constitute the right side of the foot of col. ii (now 11. 53-61), and join with the former lines 200-208 in old Frag. B. The join results in the text below. The new line numbers come first, the old line numbers follow between round brackets. These are still needed since the commentary in the previous article with this numbering needs to be used in conjunction with the newly numbered text. Line commentaries and all other references are to the new numbers. 
Here and there our earlier readings have been improved. The corrections are discussed in the line commentary, see especially 11. 59 (apallagê?), 75 (oil tax), 77

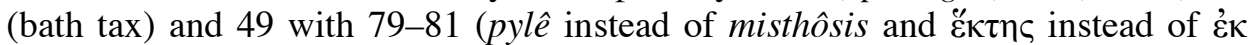
$\tau \hat{\Upsilon} \varsigma)$.

The rearrangement of the text, which overall lists inpayments to the bank over the course of a month, results in the following structure:

1-70 End of the record with details for year 13 (235/234 BC), most probably the current year, totalled in 11. 66-70. Payments are divided into those for civilians and those for the military. Two exchange rates are recorded in use for different taxes, those charged at the rate of 26.5 obols to a silver tetradrachm (e.g. 11. 6768 ) and those at 24.5 obols (1. 69), i.e. virtually at par. Payments for year 13 at the latter more favourable rate (1. 69, 8 talents $2043 \mathrm{dr} .0 .25 \mathrm{ob}$.) are more than double those at the higher rate (11. 67-68, 3 talents $5589 \mathrm{dr}$. 4.5 ob. including the agio). The rates applied are not always specified in the course of the text, perhaps because this is a summary record with more detailed records lying behind it.

Since total inpayments for year 13, including the agio, amount to 12 talents $1632 \mathrm{dr} .4 .75 \mathrm{ob}$. (paid in bronze), the $3899 \mathrm{dr} .5 .5 \mathrm{ob}$., which are recorded in 11 . $1-65$, represent only $5.3 \%$ of the total (see Table 1). A large number of columns are likely to have preceded the surviving part of the text.

Table 1. Surviving inpayments for year $13(235 / 234$ BC)

\begin{tabular}{|c|c|c|c|c|c|}
\hline Lines & Tax details & Paid by & dr. & ob. & $\underline{\text { Notes }}$ \\
\hline $1-6$ & salt tax & civilian & 826 & 4 & \\
\hline $7-15$ & & military & 54 & 3 & \\
\hline $16-21$ & guard tax & civilian & 70 & 4 & \\
\hline $22-28$ & guard tax for work- & $\begin{array}{l}\text { civilian } \\
\text { military }\end{array}$ & $\begin{array}{l}154 \\
32\end{array}$ & 3 & \\
\hline $29-37$ & pasturage tax & $\begin{array}{l}\text { civilian } \\
\text { military }\end{array}$ & $\begin{array}{l}306 \\
{[198}\end{array}$ & $\begin{array}{l}2.5 \\
3.5]\end{array}$ & \\
\hline $38-39$ & collection on geese & civilian (?) & 98 & & \\
\hline $40-44$ & tax on vineyards & civilian & 374 & 2 & \\
\hline $45-50$ & tax on orchards & civilian & 181 & 0.5 & \\
\hline $51-58$ & $\begin{array}{l}\text { guard tax on sacred } \\
\text { land (?) }\end{array}$ & $\begin{array}{l}\text { civilian } \\
\text { military }\end{array}$ & & $\begin{array}{l}? \\
2.25\end{array}$ & \\
\hline & & & 220 & 4 & in bronze \\
\hline $59-62$ & grain rents in cash & civilian & $\begin{array}{l}234 \\
91\end{array}$ & & in bronze \\
\hline$\underline{63-65}$ & bank transfers & $\underline{\text { civilian }}$ & 1038 & $\underline{0.75}$ & \\
\hline $1-65$ & surviving total & together & $\underline{3899}$ & $\underline{5.5}$ & \\
\hline
\end{tabular}

71-133 A record of arrears, divided into civilian and military payments, for taxes running back from year 12 (234/233 BC) to year 7 (241/240 BC), totalled in 11. 130-133 (see Table 2). After the fuller entry for year 12 no civilian payments 
appear to be recorded, though the status of some entries is not entirely clear. A larger proportion of arrears were paid in bronze at the higher rate of $26.5 \mathrm{ob}$. to a tetradrachm compared with the rate virtually at par, which after year 12 is not specified as paid at $24.5 \mathrm{ob}$. but simply recorded as $\chi \alpha \lambda \kappa o \hat{v}$ (in bronze), 11.108 and 132. The high level of arrears for the dyke tax paid by the military (35\% of total arrears) is striking: 100-aroura cleruchs in 11. 112 (year 10), 116 (year 9) and 126 (year 7); misthophoroi in 11. 103-104 (year 11); cf. 119-121 (year 8), unclear which group. A particularly active collector may have been in post in year 13 .

\section{Table 2. Record of arrears}

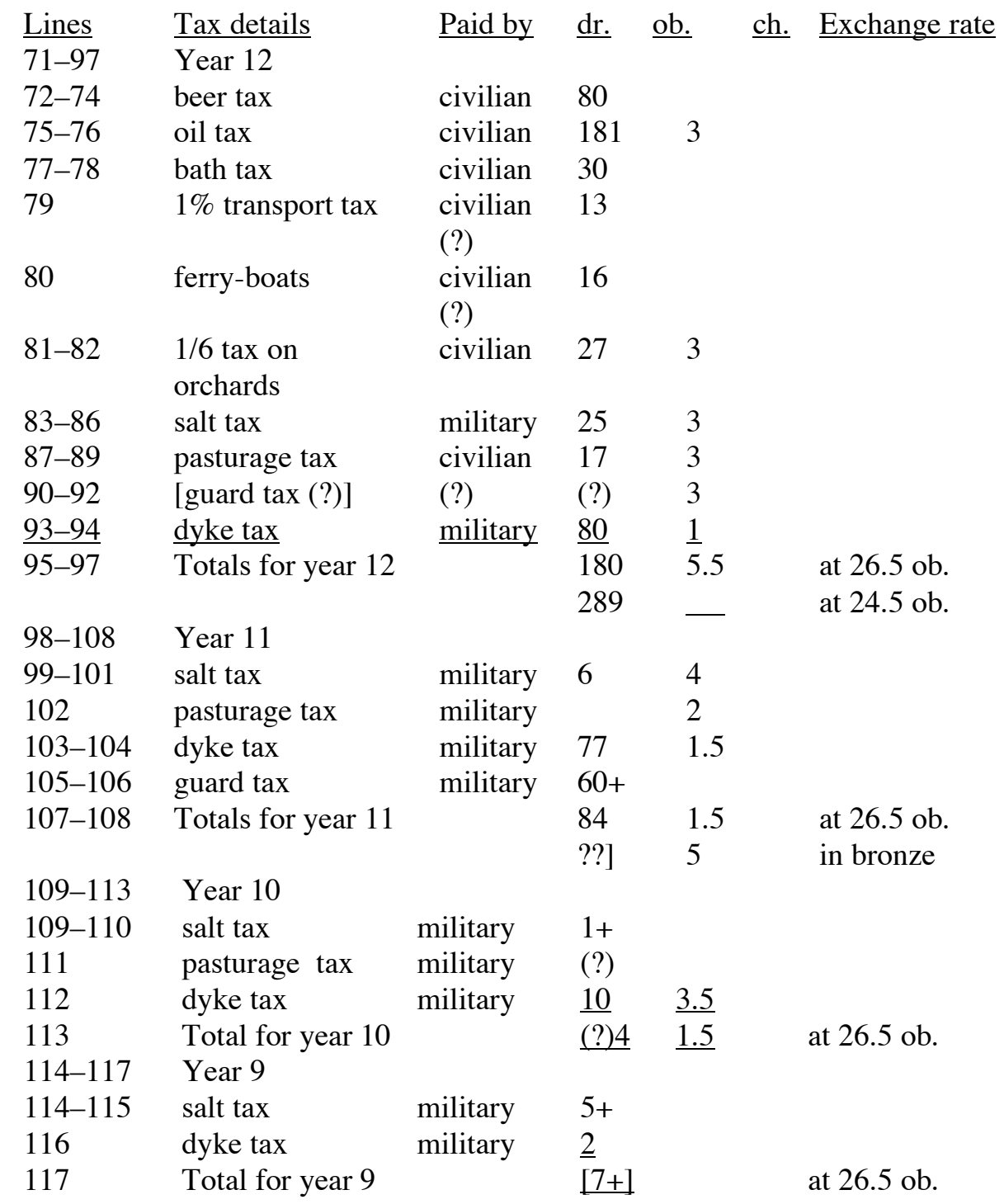




\begin{tabular}{|c|c|c|c|c|c|c|}
\hline$\underline{\text { Lines }}$ & $\underline{\text { Tax details }}$ & Paid by & dr. & ob. & ch. & Exchange rate \\
\hline$\overline{118-124}$ & Year 8 & & & & & \\
\hline 118 & salt tax & military & 3 & 3 & & \\
\hline $119-121$ & dyke tax & (?) & 75 & 1.5 & & \\
\hline $122-123$ & guard tax (land) & military & $\underline{8}$ & $\underline{3}$ & & \\
\hline 124 & Total for year 8 & & $\underline{87}$ & 1.5 & & at $26.5 \mathrm{ob}$. \\
\hline $124-127$ & Year 7 & & & & & \\
\hline $126-127$ & dyke tax & military & 15 & 4 & & \\
\hline \multirow[t]{4}{*}{$128-133$} & Composite & & $3[89]$ & [2] & & at $26.5 \mathrm{ob}$. \\
\hline & arrears [year 7] & & 40 & 5 & 1 & agio \\
\hline & up to year 12 & & $\underline{303}$ & $\underline{5}$ & $\underline{0}$ & in bronze \\
\hline & Total & & $\overline{734}$ & $\underline{\overline{0}}$ & $\underline{1}$ & \\
\hline
\end{tabular}

134-143 Details of athikta (see ed. princ., note ad. loc.), which include marriage contracts, trierarchêma and crown tax paid by the military. All payments are converted to bronze at the higher rate and add up to $81 \mathrm{dr} .3 \mathrm{ob}$. and $1 \mathrm{ch}$. (1. 143).

144-152 Record of civilian payments for the othoniêra (charged on woollen products for year 13 , cf. 1. 145) all charged at the higher conversion rate and totalling $1275 \mathrm{dr} .3 \mathrm{ob}$. (1. 152).

153-177 Payments involving the provisioning (sitarchiai, 1. 174) for military men (cleruchs, 1. 172; misthophoroi, 11. 164, 171), with payments for light structures (anastamata or bivouacs, 11. 154, 163, 170, 172) for elephant hunters (11. 157-158) and possibly other military men, for horse feed (11. 160, 173), the price (timê) of a horse (1. 161) and for crown tax (1. 171). As earlier, details for year 13 (11. 153-162) are followed by arrears: year 12 (11. 163-169), two further (illegible) years (11. 170 and 171), and year 2 (11. 172-173). A final total (1. 177) of 349 dr. 2.5 ob. 1 ch. is made up of 296 dr. 2 ob. including an agio (1l. 174-175) and $53 \mathrm{dr} .0 .5 \mathrm{ob} .1 \mathrm{ch}$. paid straightforwardly in bronze (1. 176).

178-208 The account, as it survives, concludes with a group of miscellaneous payments:

11. 178-180 tax on hides of 4 dr. $2.5 \mathrm{ob}$. including agio.

11. 181-194 details of dyke tax on sacred land for year 13 from both civilians (1l. 182-185) and military groups (11. 186-194).

11. 195-199 possibly other tax details, totalled in 11. 196-199.

11. 200-203 illegible payments, totalling more than $260 \mathrm{dr}$.

11. 205-206 a mysterious entry recording 2 talents plus more than $4000 \mathrm{dr}$.

11. 207-208 a large sum of $2990 \mathrm{dr} .3 .75 \mathrm{ob}$. for allowances (?).

We have nothing to add on the verso, which is perhaps the most intriguing part of this text. 
New measurements: $32 \times 60.5 \mathrm{~cm}$

Kolleseis at ca. 2.5, 18.5, 33.5, $51.5 \mathrm{~cm}$ from the left edge, at regular intervals of $15-16 \mathrm{~cm}$.

\section{col. i}

$$
\begin{aligned}
& 1(=148) \\
& 2(=149) \\
& 3(=150) \\
& 4(=151) \\
& 5 \text { (= 152) } \\
& 6(=153) \\
& 7 \text { (=154) } \\
& 8(=155) \\
& 9 \text { (=156) } \\
& 10 \text { (=157) } \\
& 11 \text { (= 158) } \\
& 12 \text { (= 159) } \\
& 13 \text { (= 160) } \\
& 14 \text { (= 161) } \\
& 15 \text { (= 162) } \\
& 16(=163) \\
& 17 \text { (= 164) } \\
& 18 \text { (= 165) } \\
& 19 \text { (= 166) } \\
& 20 \text { (=167) } \\
& 21 \text { (=168) } \\
& 22 \text { (= 169) } \\
& 23 \text { (= 170) } \\
& 24 \text { (= 171) } \\
& 25 \text { (=172) } \\
& 26 \text { (= 173) } \\
& 27 \text { (=174) } \\
& 28 \text { (=175) } \\
& 29 \text { (= 176) } \\
& 30 \text { (=177) } \\
& 31 \text { (=178) }
\end{aligned}
$$

one line missing?

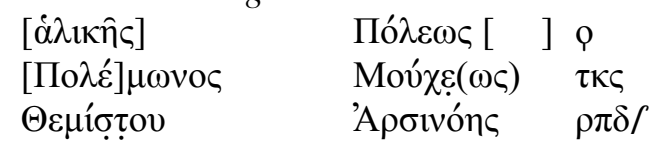

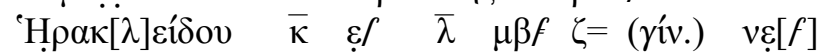

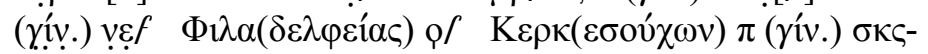

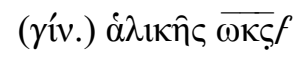

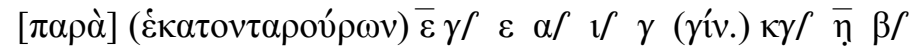
[.. ] $\gamma / \varepsilon / \zeta / \overline{i \beta} \varepsilon \overline{\mathrm{ic}} \alpha / \overline{\mathrm{i}} \beta / \delta$

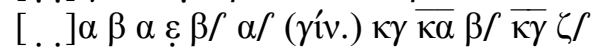

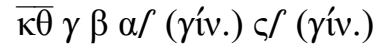

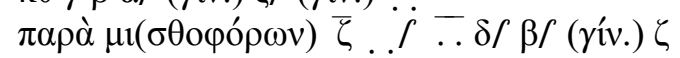

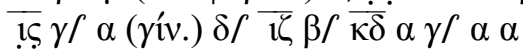

$\delta \alpha \gamma / \alpha \alpha \beta / \beta /\left(\gamma^{\prime} v.\right) \kappa \varsigma \overline{\kappa \eta ̣} \alpha \varepsilon / \alpha /\left(\gamma^{\prime} v.\right) \eta$ ( ív. $^{\prime} v \delta /$

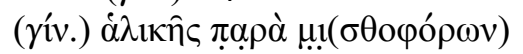

] ६. . $\mu \circ \varphi \nu \lambda \alpha \kappa \iota \tau(1 \kappa о \hat{)})$

] По́ $\varepsilon \omega \varsigma$ ह $\bar{\lambda}_{1}$

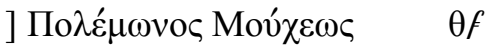

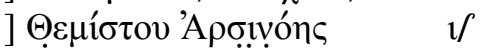

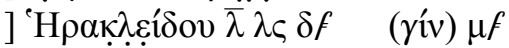

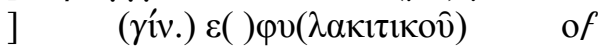

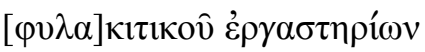

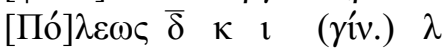

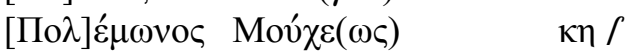

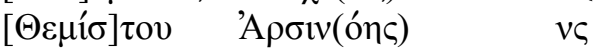

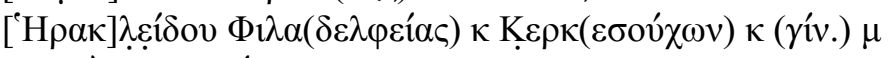
$[\pi \alpha \rho] \dot{\alpha} \mu \mathrm{i}\left(\sigma \theta 0 \varphi \rho_{0} \omega \omega\right) \quad \lambda \beta$

$$
\text { ( } \gamma^{\prime} \text { v. } \rho \pi \varsigma /
$$

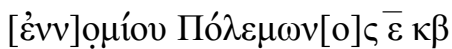

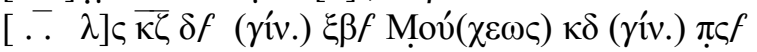

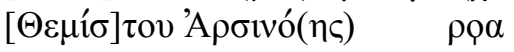


col. ii

$32(=179)$

$33(=180)$

$34(=181)$

$35(=182)$

$36(=183)$

$37(=184)$

$38(=185)$

$39(=186)$

$40(=187)$

41 (= 188)

$42(=189)$

$43(=190)$

$44(=191)$

$45(=192)$

46 (= 193)

$47(=194)$

48 (=195)

49 (= 196)

50 (= 197)

51 (= 198)

$52(=199)$

$53(=200+1)$

$54(=201+2)$

$55(=202+3)$

$56(=203+4)$

$57(=204+5)$

$58(=205+6)$

$59(=206)$

$60(=207)$

$61(=208+7)$

62 (=209)

\section{col. iii}

$63(=8)$

64 (=9)

$65(=10)$

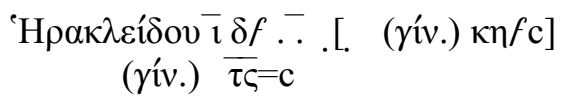

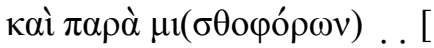

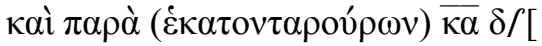

$\overline{\kappa \beta} \quad \rho \gamma=\overline{\kappa \gamma} \varepsilon f \quad \overline{\kappa \theta}[$

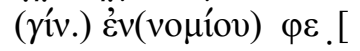

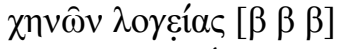

$\beta \beta \beta \beta$ ( $\left.\gamma^{\prime} v.\right)$ i $\delta \lambda \mathrm{o}\left(\gamma \varepsilon^{\prime} \alpha \varsigma\right)$ ọ

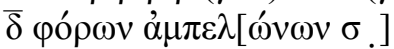

$\bar{\zeta} \quad \lambda \eta \quad\left(\gamma^{\prime} v.\right) \quad \sigma \lambda \eta$. [

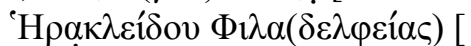

$\Theta \varepsilon \mu i ́ \sigma \tau o v \quad \bar{\lambda}[$

( $\left.\gamma^{\prime} \mathrm{v}.\right) \quad \tau \mathrm{o} \delta=[$

$\pi \alpha \rho \alpha \delta \varepsilon i ́ \sigma \omega v . .[$

$\overline{\kappa .} \delta / \overline{\kappa .} \varepsilon \frac{. \bar{\kappa} \bar{\kappa}}{.}[$

$\Theta \varepsilon \mu i ́ \sigma \tau o v \overline{\varepsilon \varepsilon}$.[

${ }^{\circ} \mathrm{H} \rho \alpha \kappa \lambda \varepsilon i ́ \delta$ ov $\bar{i} \mathrm{v}$. [

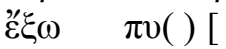

( $\gamma^{\prime} v$.) $\rho \pi \alpha \mathrm{c}[$

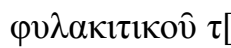

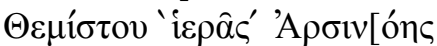

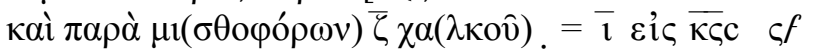

$\bar{\kappa} \xi_{\varsigma} / \chi(\alpha \lambda \kappa о \hat{\imath}) \gamma \bar{\lambda} \mathrm{\imath} / \mathrm{c} \ldots . . . \mathrm{l}-\mathrm{c}$

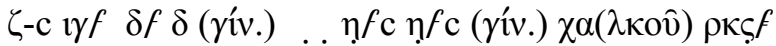

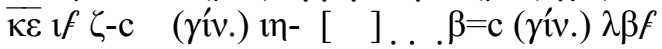

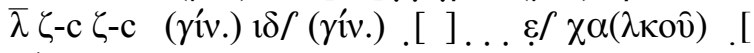

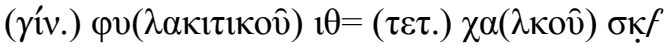

$\dot{\alpha} \pi \alpha \lambda \alpha \gamma \underline{\emptyset} \varsigma$

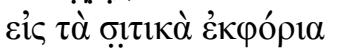

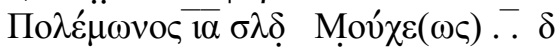

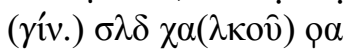

three or four lines missing

$\delta]$ ] $\alpha \gamma_{\rho} \alpha \varphi \hat{\omega} \mathrm{y}$

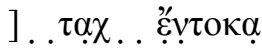

] $\bar{\zeta} \chi \alpha(\lambda \kappa о \hat{)})$ 'A $\lambda \eta c(\tau \varepsilon \tau$. 
W. Clarysse, D.J. Thompson and L. Capron, An early Ptolemaic bank register

$66(=11)$
$67(=12)$
$68(=13)$
$69=14)$
$70(=15)$
$71=16)$
$72(=17)$
$73(=18)$
$74(=220+19)$
$75(=221+20)$
$76=222+21)$
$77(=223+22)$
$78(=23+224+210)$
$79(=24+211)$
$80(=25+212)$
$81(=26+213)$
$82(=27)$
$83(=28)$
$84(=29)$
$85(=30)$
$86=31)$
$87(=32)$
$88(=33)$
$89=34)$

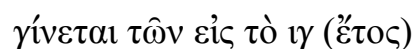

$\chi \alpha(\lambda \kappa o \hat{)}) \varepsilon i \varsigma \overline{\kappa \varsigma} \mathrm{c}$

$\dot{\varepsilon} \pi \alpha \lambda \lambda \alpha \gamma \grave{n}$

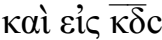

$\tau \alpha(\lambda.) \gamma \gamma^{\prime} \Gamma \tau \xi \gamma-$

'B $\sigma \kappa \varsigma / \mathrm{c}$

$\tau \alpha(\lambda.) \eta{ }^{\prime} \mathrm{B} \mu \gamma(\tau \varepsilon \tau$.

(yív.) $\chi \alpha \lambda \kappa o \hat{~}$

$\tau \alpha(\lambda). i \beta{ }^{\prime} \mathrm{A} \chi \lambda \beta \beta c(\tau \varepsilon \tau$.

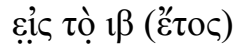

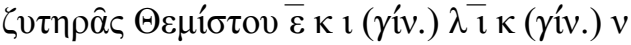

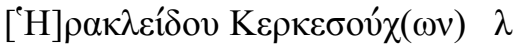

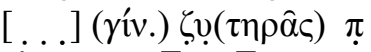

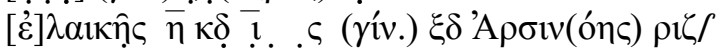

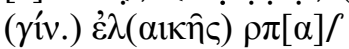

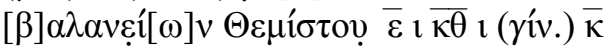

$\ldots \ldots \ldots \quad l \quad\left(\gamma^{\prime} v.\right) \lambda$

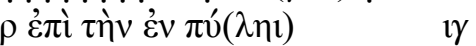

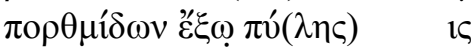

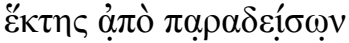

Под $\varepsilon^{\prime} \mu \omega v o \varsigma \kappa \zeta /$

$\dot{\alpha} \pi \circ \gamma \rho \alpha \varphi \hat{\omega} v \dot{\alpha} \lambda \omega \kappa \hat{\varphi} \varphi$

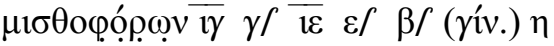

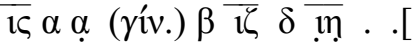

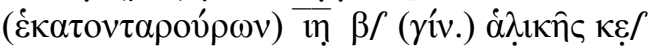

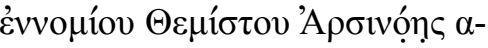

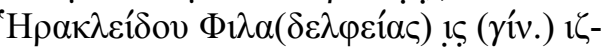

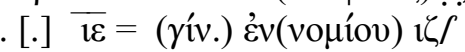

\section{col. iv}

$90(=35)$
$91(=36)$
$92(=37)$
$93(=38)$
$94(=39)$
$95(=40)$
$96(=41)$
$97(=42)$

$98 \quad(=43)$

$99 \quad(=44)$

$100(=45)$

$101(=46)$

$102(=47)$

$103(=48)$ about 2 lines missing

$$
\begin{aligned}
& \text { [ ] ]. } \bar{\kappa} \zeta \quad \alpha-c
\end{aligned}
$$

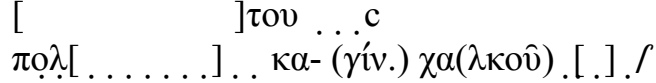

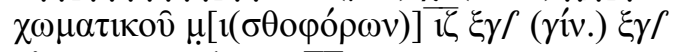

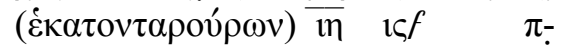

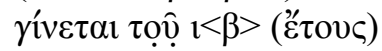

$$
\begin{aligned}
& \begin{array}{ll}
\chi \alpha \lambda \kappa \mathrm{o} \hat{v} \varepsilon i \varsigma \overline{\kappa \varsigma} \mathrm{c} & \rho \pi f \mathrm{c} \\
\varepsilon i \bar{\kappa} \bar{\kappa} \mathrm{c} & \sigma \pi \theta
\end{array}
\end{aligned}
$$

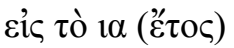

$\dot{\alpha} \pi \circ \gamma \rho \alpha \varphi \hat{\omega} v \dot{\alpha} \lambda \iota \kappa \hat{\omega} v \mu\left[1\left(\sigma \theta 0 \varphi \rho_{\rho} \omega v\right)\right] \bar{\gamma} \gamma /$

$$
\begin{aligned}
& \text { is } \beta / \\
& \left(\gamma^{\prime} \mathrm{iv} .\right) \varsigma \\
& (\dot{\varepsilon} \kappa \alpha \tau o v \tau \alpha \rho o v \rho \omega v) \overline{\mathrm{m}}=\overline{\kappa \gamma}=\left(\gamma^{\prime} \mathbf{v} .\right) f\left(\gamma^{\prime} \mathrm{v} .\right) \varsigma f \\
& \dot{\varepsilon} v v o \mu \text { íov }(\dot{\varepsilon} \kappa \alpha \tau o v \tau \alpha \rho \circ o v \rho \omega) \bar{\varepsilon}= \\
& \chi \omega \mu \alpha \tau \text { }
\end{aligned}
$$


$104(=49)$

$105(=50)$

$106(=51)$

$107(=52)$

$108(=53)$

$109(=54)$

$110(=210)$

$111(=211)$

$112(=212)$

$113(=213+225)$

$114(=226)$

$115(=227)$

$116(=228)$

$117(=56+229)$

$118(=57+230)$

$119(=58+231)$

$120(=59)$

\section{col. $\mathbf{v}$}

$121(=60)$

$122(=61)$

$123(=62)$

$124(=63)$

$125(=64)$

$126(=65)$

$127(=66)$

$128(=67)$

$129(=68)$

$130(=69)$

$131(=70)$

$132(=71)$

133 (= 72$)$

$134(=73)$

$135(=74)$

$136(=75)$

$137(=76)$

$138(=77)$

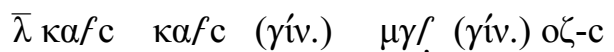

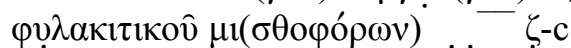
$\zeta$-c $\left(\gamma^{\prime} \mathbf{v}.\right) 1 \delta / \quad\left(\gamma^{\prime} v.\right) \xi[]$.

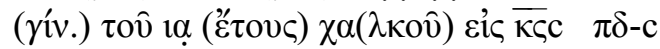
$\chi \alpha \lambda \kappa \circ \hat{\text { [ }}] . \digamma=$

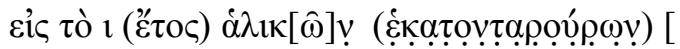

$\mu \mathrm{t}\left(\sigma \theta 0 \varphi \rho_{\rho} \rho \omega v\right) \overline{i \varepsilon} \alpha\left(\gamma^{\prime} v.\right)$ [

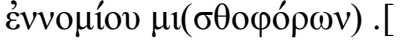

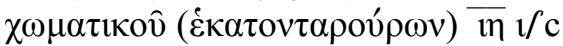

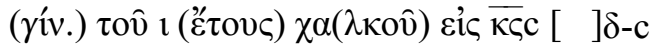

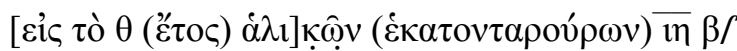
.. $\div \beta /$. ].

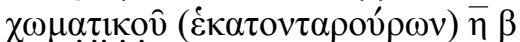

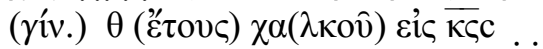

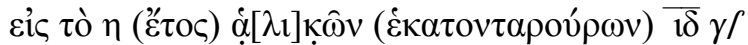
$\chi \omega \mu \alpha \tau$ $\bar{\kappa} \delta \rho f \mathrm{c} \overline{\kappa \alpha} \varepsilon f \mathrm{c}\left(\gamma^{\prime} \mathrm{v}.\right) .$.

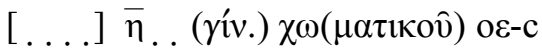

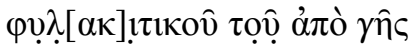
$\mu \mathrm{l}(\sigma \theta$ о

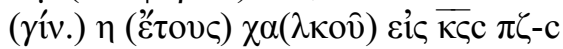

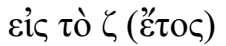

$\chi \omega \mu \alpha \tau \iota \kappa о \hat{~(\varepsilon ́ \kappa \alpha \tau о v \tau \alpha \rho о и ́ \rho \omega v) ~} \bar{\eta} \delta$ $1 \alpha f$ ( $\left.\gamma^{\prime} \mathbf{v}.\right) i \varepsilon f$

$[\ldots . . . \tau \ldots$

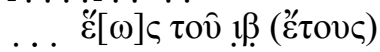

$\chi \alpha \lambda \kappa \circ \hat{v} \varepsilon i \varsigma \bar{\kappa} \varsigma \mathrm{c}$

$\dot{\varepsilon} \pi \alpha \lambda \alpha \gamma \grave{\eta}$

$\chi \lambda$ коиิ

$\tau[\pi \theta=]$

$\mu f \chi \alpha(\lambda \kappa о \hat{\varsigma} \varsigma)$

$\tau \gamma f$

( $\left.\gamma^{\prime} \dot{v}.\right) \psi \lambda \delta \chi \alpha(\lambda \kappa \circ \hat{\varsigma} \varsigma)$

$\dot{\alpha} \theta \dot{\kappa} \kappa \tau \omega v$

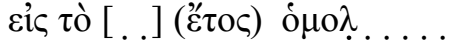

$\bar{\zeta} \overline{\bar{\zeta}} \kappa$

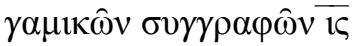

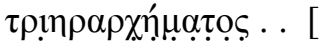

( $\left.\gamma^{\prime} v.\right) \mu$

$\kappa$ 
W. Clarysse, D.J. Thompson and L. Capron, An early Ptolemaic bank register

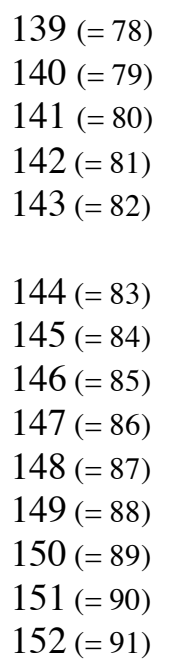

\section{col. vi}

$153(=214+92)$

$154(=215+93)$

$155(=216+94)$

156 (=95)

$157(=217+96)$

$158(=218+97)$

$159(=219+98)$

$160(=99)$

$161(=100)$

$162(=101)$

$163(=102)$

$164(=103)$

$165(=104)$

166-167

168 (= 107)

$169(=108)$

170 (= 109)

$171(=110)$

$172(=111)$

$173(=112)$

$174(=113)$

175 (= 114)

176 (=115) $\overline{\kappa \beta} \gamma$

( $\gamma^{\prime} v$. $) \xi \gamma$

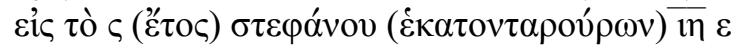

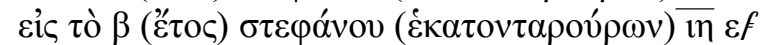

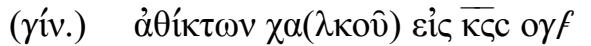

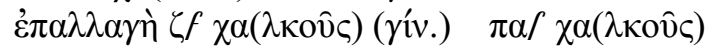

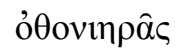

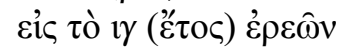

Пó $\lambda \varepsilon \omega \varsigma \quad \overline{\imath \beta} \quad \tau \delta$

Поде́ $\mu \omega v$ о $\quad \overline{i \beta} \quad$ бо५-

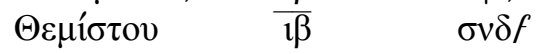

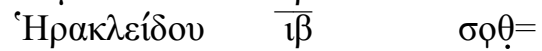

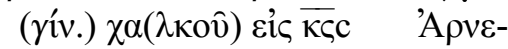

$\dot{\varepsilon} \pi \alpha \lambda \lambda \alpha \gamma \grave{\eta} \quad \rho \kappa=$

( $\gamma^{\prime}$ v. ${ }^{\prime} \mathrm{A} \sigma 0 \varepsilon /$

one or two lines missing

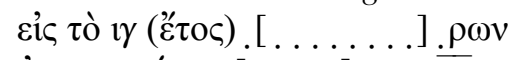

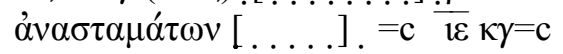

$\overline{i c} \ldots[\quad] i \xi \kappa \eta=\left(\gamma^{\prime} v.\right)$ [. . ]

[ ] $] \rho / \mathrm{c}$

ко .] ]

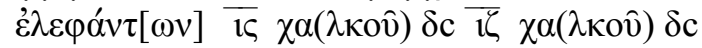

$\overline{\kappa \eta} \chi \alpha(\lambda \kappa \circ \hat{v}) \delta \mathrm{c}[$

[i] $\pi \pi \circ \mathrm{ov} \tau \rho \circ \varphi \hat{\eta} \varsigma$

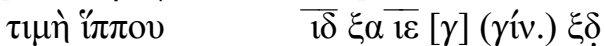

( $\left.\gamma^{\prime} \dot{v}.\right) \chi \alpha(\lambda \kappa o \hat{)}) \varepsilon i \varsigma \overline{\kappa \varsigma} \mathrm{c} \sigma \lambda \eta-(\tau \varepsilon \tau.) \chi \alpha(\lambda \kappa \circ 0 \hat{)}) . .=\mathrm{c}$

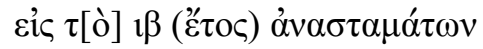

$\pi \alpha \rho \grave{\alpha} \mu 1\left(\sigma \theta 0 \varphi \rho_{\rho} \omega v\right) \overline{\imath \varepsilon} \eta / \mathrm{c} \delta\left(\gamma^{\prime} v\right.$.) $\beta / \mathrm{c} \overline{\imath \zeta}$.[. . .] . .

$\overline{\kappa \alpha} \alpha=(\tau \varepsilon \tau) \quad.\left(\gamma^{\prime} v.\right) i \zeta / \mathrm{c} \chi \alpha(\lambda \kappa o \hat{v}) .[$

two lines illegible

$\kappa \ldots \ldots \tau \ldots \kappa \bar{\varepsilon} \alpha[$

one line illegible

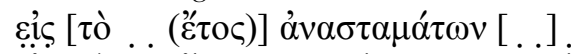

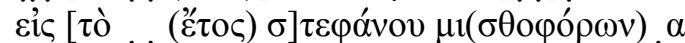

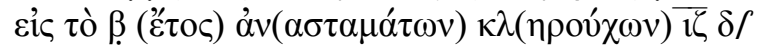

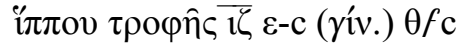

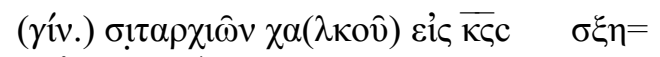
$\dot{\varepsilon} \pi \alpha \lambda \lambda \alpha(\gamma \grave{\eta})$

$\alpha \lambda \kappa о \hat{v}$

$\kappa \eta$

vүc $\chi$ 
$178(=117)$

$179(=118)$

$180(=119)$

$181(=120)$

$182(=121)$

$183(=122)$

col. vii

$184(=123)$

$185(=124)$

$186(=125)$

$187(=126)$

$188(=127)$

$189(=128)$

$190(=129)$

$191(=130)$

$192(=131)$

$193(=132)$

$194(=133)$

$195(=134)$

$196(=135)$

$197(=136)$

$198(=137)$

$199(=138)$

$200(=139)$

$201(=140)$

$202(=141)$

$203(=142)$

$204(=143)$

$205(=144)$

$206(=145)$

$207(=146)$

$208(=147)$

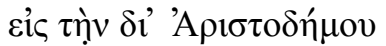

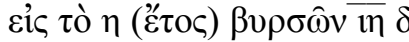

$\dot{\varepsilon} \pi \alpha \lambda \lambda \alpha(\gamma \grave{\eta})=\mathrm{c}\left(\gamma^{\prime} \mathbf{v}.\right) \delta=\mathrm{c}$

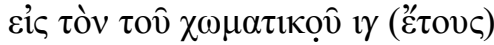

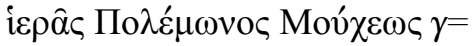

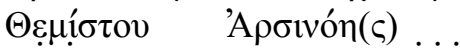

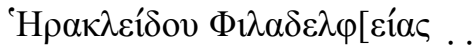
( $\gamma^{\prime} \mathrm{i}$.) in

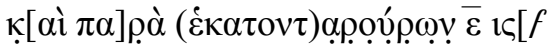

ıৎf ( $\gamma^{\prime} \mathbf{i} v$.) $\pi \gamma=\ldots[$

$\overline{\mathrm{i}} \mathrm{icf} \overline{\mathrm{ic}} \mathrm{\imath} \varsigma \mathrm{f} \overline{\mathrm{m}}[\mathrm{i} \varsigma f$

illegible

[ ] $] \overline{\kappa \varepsilon} \imath \varsigma[f$

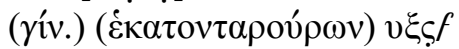

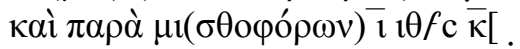
$\lambda \beta f \mathrm{c} \imath \theta f(\tau \varepsilon \tau$.) $\kappa \alpha f \mathrm{c} v \delta-$ $\alpha=v \varepsilon f \mathrm{c} \kappa \varsigma \kappa \varsigma\left(\gamma^{\prime}{ }^{\prime} v.\right) \tau[$ [..]. $\mathrm{v}[$ about ten lines lost $\dot{\varepsilon} \pi \alpha \lambda \lambda \alpha \gamma \grave{n}[$

$\chi \alpha \lambda \kappa о \hat{~[~}$

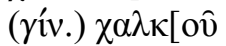

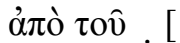

. $\alpha . \tau \alpha[$

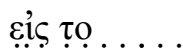

$\bar{\varepsilon} \ldots \bar{\imath} \gamma$

$\propto \kappa \kappa(\gamma i ́ v.) \sigma \xi$. [

eis $\tau \mathrm{o} . . \tau[$

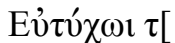

$\overline{\kappa \alpha}\left(\tau \alpha \alpha^{\prime} \lambda.\right) \beta^{\prime} \Delta[$

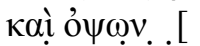

$\bar{\gamma}{ }^{\prime} \mathrm{B} \lambda \phi / \mathrm{c}(\tau \varepsilon \tau$. 
Verso (in a different hand on the back of col. $i$ )

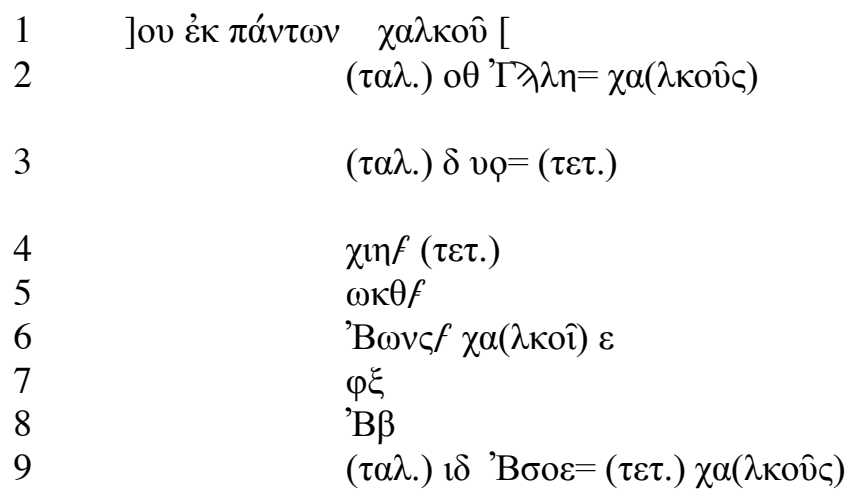

\section{Translation}

\section{col. i}

$1(=148) \quad$ [for the salt tax]

$2(=149) \quad$ [for the meris of Pole]mon

$3(=150) \quad$ [for the meris of Them] istos

$4(=151)$

$5(=152)$

$6(=153)$

$7(=154)$

$8(=155)$

$9(=156)$

$10(=157)$

$11(=158)$

$12(=159)$

$13(=160)$

$14(=161)$

$15(=162)$ 80 dr., total 226 dr. 1 ob. 2 dr. 3 ob., total 7 total $54 \mathrm{dr} .3 \mathrm{ob}$. for the city $90 \mathrm{dr}$.

at Mouchis $326 \mathrm{dr}$.

at Arsinoe $184 \mathrm{dr} .3 \mathrm{ob}$. for the meris of Herakleides, day 20: 5 dr. 3 ob., day 30: $42 \mathrm{dr}$. 5 ob., 7 dr. 2 ob., total 55 dr. 4 ob.

total 55 dr. 4 ob. at Philad(elpheia) 90 dr. 3 ob. at Kerke(soucha)

total for salt tax $826 \mathrm{dr} .4 \mathrm{ob}$.

[from] the 100-aroura men, day 5: 3 dr. 3 ob., 5 dr., 1 dr. 3 ob., 10 dr. 3 ob., 3 dr., total 23 dr. 3 ob., day 8: 2 dr. 3 ob.

[ $\quad$ ] 3 dr. 3 ob., 5 dr. 3 ob., 7 dr. 3 ob., day 12: 5 dr., day 16: 1 dr. 3 ob., day 17: 2 dr. 3 ob., 4 dr.

[ $\quad$ ] 1 dr., 2 dr., 1 dr., 5 dr., 2 dr. 3 ob., 1 dr. 3 ob., total 23 dr., day 21: 2 dr. 3 ob., day 23: 7 dr. 3 ob.

day 29: 3 dr., 2 dr., 1 dr. 3 ob., total 6 dr. 3 ob., total . .

from the professional soldiers, day 7: . . 3 ob., day . : 4 dr. 3 ob.,

day 16: 3 dr. 3 ob., 1 dr., total 4 dr. 3 ob., day 17: 2 dr. 3 ob., day 24: 1 dr., 3 dr. 3 ob., 1 dr., 1 dr.

4 dr., 1 dr., 3 dr. 3 ob., 1 dr., 1 dr., 2 dr. 3 ob., 2 dr. 3 ob., total 26, day $28: 1$ dr., 5 dr. 3 ob., 1 dr. 3 ob., total 8 dr.

total for salt tax from the professional soldiers 
$16(=163) \quad$ for $\ldots$ guard tax

17 (=164) for the city, day 30

$10 \mathrm{dr}$.

$18(=165)$

for the meris of Polemon at Mouchis

9 dr. 5 ob.

$19(=166)$

for the meris of Themistos at Arsinoe

10 dr. 3 ob.

$20(=167)$

for the meris of Herakleides, day 30: $36 \mathrm{dr}$., $4 \mathrm{dr}$. 5 ob., total $40 \mathrm{dr}$. 5 ob.

$21(=168)$

]

total for ... guard tax

70 dr. 4 ob.

$22(=169)$

for guard tax for the workshops

$23(=170)$

for the city, day 4: $20 \mathrm{dr}$., $10 \mathrm{dr}$., total $30 \mathrm{dr}$.

$24(=171)$ for the meris of Polemon at Mouchis

28 dr. 3 ob.

$25(=172)$ for the meris of Themistos at Arsinoe

$56 \mathrm{dr}$.

$26(=173)$ for the meris of Herakleides, at Phila(delpheia) 20 dr., at Kerk(esoucha) $20 \mathrm{dr}$., total $40 \mathrm{dr}$.

$27(=174)$

$28(=175)$ from the] professional soldiers $32 \mathrm{dr}$.

$29(=176) \quad$ for pasturage tax for the meris of Polemon, day 5: $22 \mathrm{dr}$.

30 (=177) [day 00: 3] 6 dr., day 27: 4 dr. 4 ob., total 62 dr. 4 ob., at Mouchis 24 dr., total 86 dr. 4 ob.

$31(=178)$ for the meris of Themistos at Arsinoe

$191 \mathrm{dr}$

col. ii

$32(=179)$

for the meris of Herakleides, day 10: 4 dr. 4 ob., day 00: [ . . dr.

$33(=180)$ ob., total $28 \mathrm{dr} .4 .5 \mathrm{ob}$.]

$34(=181) \quad$ and from the professional soldiers [

35 (= 182) and from the 100-aroura men, day $21: 4 \mathrm{dr} .3 \mathrm{ob}$. [

$36(=183)$ day 22: 103 dr. 2 ob., day 23: 5 dr. 4 ob., day 29: [

$37(=184)$ total for pasturage tax $505 \mathrm{dr}$. . [

$38(=185)$

for tax collection on geese [ $2 \mathrm{dr} ., 2 \mathrm{dr} ., 2 \mathrm{dr}$.]

$39(=186)$

$2 \mathrm{dr}$., $2 \mathrm{dr}$., $2 \mathrm{dr}$., $2 \mathrm{dr}$., total $14 \mathrm{dr}$., for the collection (?) $98 \mathrm{dr}$.

$40(=187)$

$41(=188)$ day 4: for taxes on viney[ards $200 \mathrm{dr}$.]

$42(=189)$

$43(=190)$ day 7: $38 \mathrm{dr}$., total $238 \mathrm{dr}$. . [ for the meris of Herakleides at Phila(delpheia) [

$44(=191)$ for the meris of Themistos, day 30: [

$45(=192)$

total 374 dr. 2 ob. [

$46(=193)$ for (taxes on) orchards . . [

$47(=194)$ day 20+: 4 dr. 3 ob., day 20+: 5 dr., day 27: [

$48(=195)$

for the meris of Themistos, day 15: [

for the meris of Herakleides, day 12: 50+[ 
W. Clarysse, D.J. Thompson and L. Capron, An early Ptolemaic bank register

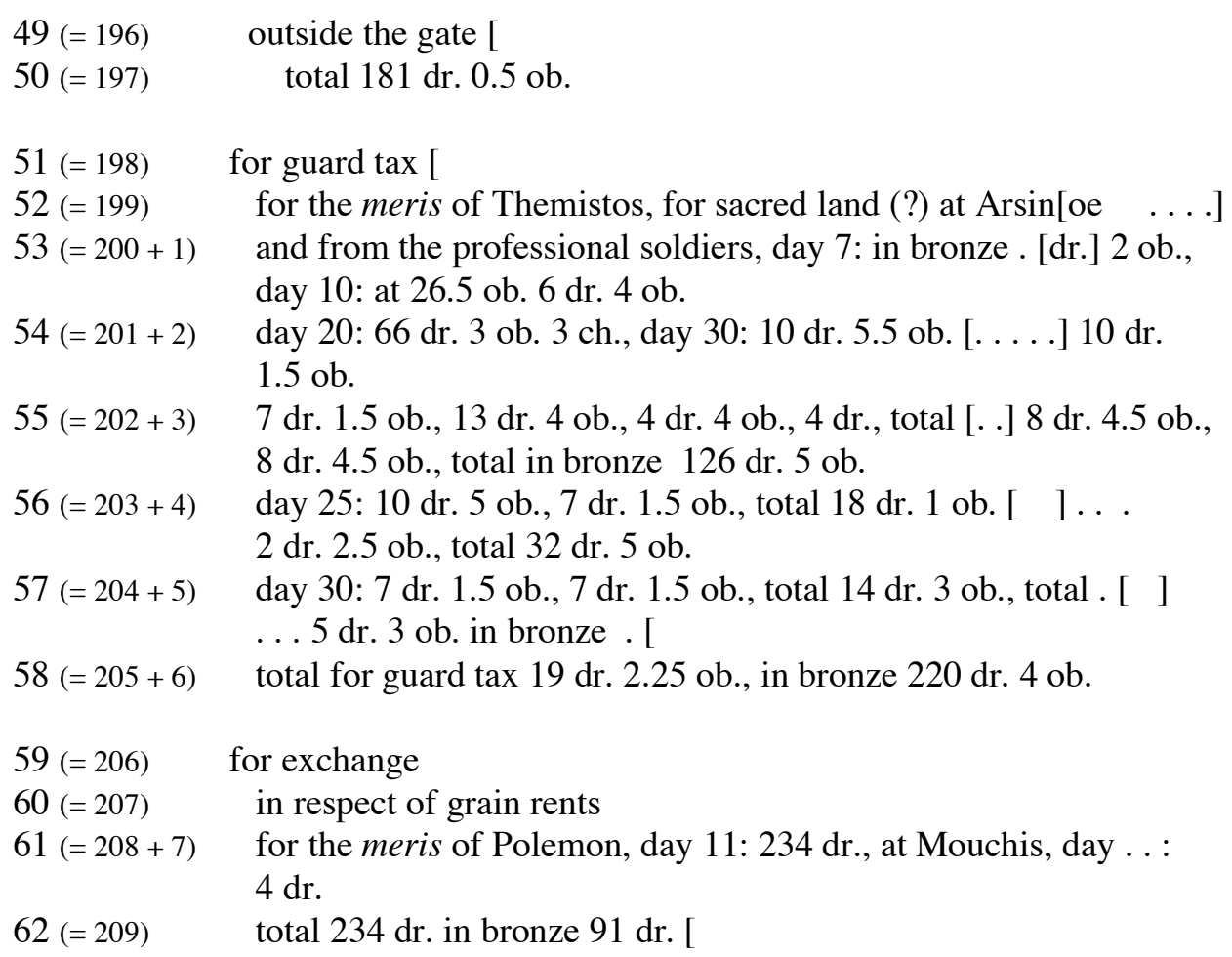

col. iii

\begin{tabular}{|c|c|}
\hline & three or four lines missing \\
\hline $63(=8)$ & [ ] for bank transfers \\
\hline $64(=9)$ & ]. . . . . . with interest (?) \\
\hline $65(=10)$ & ] day 17 in bronze $\quad 1038$ dr. $0.75 \mathrm{ob}$. \\
\hline $66(=11)$ & total of (payments) for the $13^{\text {th }}$ year \\
\hline $67(=12)$ & in bronze at 26.5 ob. $\quad 3$ ta(l.) $3363 \mathrm{dr} .1$ ob. \\
\hline $68(=13)$ & 2226 dr. 3.5 ob. \\
\hline $69(=14)$ & 8 ta(1.) 2043 dr. 0.25 ob. \\
\hline $70(=15)$ & 12 ta(l.) 1632 dr. $4.75 \mathrm{ob}$ \\
\hline $71(=16)$ & for the $12^{\text {th }}$ year \\
\hline $72(=17)$ & $\begin{array}{l}\text { for beer tax, for the meris of Themistos, day } 5: 20 \mathrm{dr} \text {., } 10 \mathrm{dr} \text {., total } \\
30 \mathrm{dr} \text {., day 10: } 20 \mathrm{dr} \text {., total } 50 \mathrm{dr} \text {. }\end{array}$ \\
\hline $73(=18)$ & for the meris of Herakleides at Kerkesoucha $30 \mathrm{dr}$. \\
\hline $74(=220+19)$ & [ ] total for beer tax $80 \mathrm{dr}$ \\
\hline $75(=221+20)$ & $\begin{array}{l}\text { for oil tax, day 8: } 24 \text { dr., day 10+: . } 6 \text { dr., total } 64 \text { dr., at Arsin(oe) } \\
117 \text { dr. } 3 \text { ob. }\end{array}$ \\
\hline$(=222+21)$ & total for oil tax $181 \mathrm{dr} .3 \mathrm{ob}$ \\
\hline
\end{tabular}


$77(=223+22)$ for bath tax for the meris of Themistos, day 5: $10 \mathrm{dr}$., day 29:10 dr., total $20 \mathrm{dr}$.

$78(=23+210+224) \ldots \ldots \ldots \quad 10 \mathrm{dr}$. total $30 \mathrm{dr}$.

$79(=24+211) 1 \%$ tax at the (bank (?)) inside the gate $13 \mathrm{dr}$.

$80(=25+212)$ for ferry-boats outside the gate $16 \mathrm{dr}$.

$81(=26+213)$ for the one sixth tax on orchards

$82(=27) \quad$ for the meris of Polemon 27 dr. $3 \mathrm{ob}$.

$83(=28) \quad$ for declarations of salt tax

$84(=29) \quad$ from the professional soldiers, day 13: $3 \mathrm{dr} .3$ ob., day 15: 5 dr. 3 ob., 2 dr. 3 ob., total $8 \mathrm{dr}$.

$85(=30) \quad$ day $16: 1 \mathrm{dr} ., 1 \mathrm{dr}$., total $2 \mathrm{dr}$., day $17: 4 \mathrm{dr}$., day $18: .$. [

86 (=31) from the 100-aroura men, day 18: $2 \mathrm{dr} .3 \mathrm{ob}$., total for the salt tax 25 dr. 3 ob.

$87(=32) \quad$ for pasturage tax, for the meris of Themistos at Arsinoe $1 \mathrm{dr} .1 \mathrm{ob}$.

$88(=33) \quad$ for the meris of Herakleides at Philadelpheia $16 \mathrm{dr}$., total $17 \mathrm{dr} .1 \mathrm{ob}$.

$89(=34) \quad$. [ . ] day 15: $2 \mathrm{ob}$., total for pasturage tax $17 \mathrm{dr} .3 \mathrm{ob}$.

col. iv

$90(=35)$

$91(=36)$

$92(=37)$

$93(=38)$

$94(=39)$

$95(=40)$

$96(=41)$

$97(=42)$

$98(=43)$

$99(=44)$

$100(=45)$

$101(=46)$

$102(=47)$

$103(=48)$

$104(=49)$

$105(=50)$

$106(=51)$ about 2 lines missing

[

] day $27: 1 \mathrm{dr} .1 .5 \mathrm{ob}$.

$$
\text { ]. . . . } 0.5 \mathrm{ob} \text {. }
$$

... [ ] . $21 \mathrm{dr} .1 \mathrm{ob}$, total in bronze . [ ] . $3 \mathrm{ob}$.

for dyke tax from the professional soldiers, day 17: $63 \mathrm{dr} .3 \mathrm{ob}$, total $63 \mathrm{dr} .3 \mathrm{ob}$.

from the 100-aroura men, day 18: $16 \mathrm{dr} .4 \mathrm{ob},, 80 \mathrm{dr} .1 \mathrm{ob}$. total for the $1<2>$ th year in bronze at $26.5 \mathrm{ob}$.

180 dr. 5.5 ob.

at $24.5 \mathrm{ob}$.

$289 \mathrm{dr}$.

for the $11^{\text {th }}$ year

for declarations of salt tax from the professional soldiers, day 13: 3 dr. 3 ob.

day 16: 2 dr. $3 \mathrm{ob}$ t total $6 \mathrm{dr}$.

from the 100-aroura men, day 18: 2 ob., day 23: 2 ob., total 4 ob., total $6 \mathrm{dr} .4 \mathrm{ob}$.

for pasturage tax from the 100-aroura men, day 5: $2 \mathrm{ob}$.

for dyke tax from the professional soldiers, day 7: $1 \mathrm{dr}$., day 21:

$32 \mathrm{dr} .4 .5 \mathrm{ob}$.

day 30: 21 dr. 4.5 ob., 21 dr. 4.5 ob., total 43 dr. 3 ob. ., total $77 \mathrm{dr} .1 .5 \mathrm{ob}$.

for guard tax from the professional soldiers . . day . . : $7 \mathrm{dr}$. $1.5 \mathrm{ob}$.

7 dr. 1.5 ob., total 14 dr. 3 ob., total 60 dr. [ 
W. Clarysse, D.J. Thompson and L. Capron, An early Ptolemaic bank register

$107(=52) \quad$ total for the $11^{\text {th }}$ year in bronze at $26.5 \mathrm{ob} .84 \mathrm{dr} .1 .5 \mathrm{ob}$.

$108(=53)$ in bronze [ ]. $5 \mathrm{ob}$.

$109(=54) \quad$ for the $10^{\text {th }}$ year for salt tax from the 100-aroura men [

$110(=210) \quad$ from the professional soldiers, day 15: $1 \mathrm{dr}$., total [

$111(=211) \quad$ for pasturage tax from the professional soldiers . [

$112(=212) \quad$ for dyke tax from the 100-aroura men, day 18: $10 \mathrm{dr} .3 .5 \mathrm{ob}$.

$113(=213+225)$ total for the $10^{\text {th }}$ year in bronze at $26.5 \mathrm{ob}$. [ ] $4 \mathrm{dr}$. $1.5 \mathrm{ob}$.

$114(=226) \quad\left[\right.$ for the $9^{\text {th }}$ year for salt] tax from the 100 -aroura men, day 18 : 2 dr. 3 ob.

115 (=227) . . day . .: 2 dr. 3 ob. . [ ] .

$116(=228) \quad$ for dyke tax from the 100-aroura men, day 8: $2 \mathrm{dr}$.

$117(=56+229)$ total for the $9^{\text {th }}$ year in bronze at $26.5 \mathrm{ob} . .$.

$118(=57+230)$ for the $8^{\text {th }}$ year for salt tax from the 100 -ar. men, day $14: 3 \mathrm{dr} .3 \mathrm{ob}$.

$119(=58+231)$ for dyke tax . [ ]. $5.5 \mathrm{ob}$.

120 (=59) day 20: 4 dr. 4.5 ob., day $21: 5$ dr. 4.5 ob., total . . .

\section{col. $\mathbf{v}$}

$121(=60) \quad[\ldots]$ day $8: .$. , total for dyke tax $75 \mathrm{dr} .1 .5 \mathrm{ob}$.

$122(=61) \quad$ for guard tax from land

$123(=62) \quad$ from the professional soldiers, day 14: $8 \mathrm{dr} .3 \mathrm{ob}$.

$124(=63) \quad$ total for the $8^{\text {th }}$ year in bronze at $26.5 \mathrm{ob} .87 \mathrm{dr} .1 .5 \mathrm{ob}$.

$125(=64) \quad$ for the $7^{\text {th }}$ year

$126(=65) \quad$ for dyke tax from the 100-aroura men, day 18: $4 \mathrm{dr}$.

127 (= 66) 11 dr. 4 ob., total 15 dr. 4 ob.

$128(=67) \quad[\ldots] \ldots$

$129(=68) \quad \ldots$ up to the $12^{\text {th }}$ year

$130(=69) \quad$ in bronze at $26.5 \mathrm{ob}$

$131(=70)$

$132(=71)$

$133(=72)$

agio

in bronze

$3[89$ dr. 2 ob.]

40 dr. 5 ob. 1 ch.

total

303 dr. 5 ob.

734 dr. 1 ch.

$134(=73) \quad$ for "untouched"

$135(=74) \quad$ for the $\left[.^{\text {th }}\right]$ year $\ldots \ldots \ldots$

$136(=75) \quad$ day $7: 20 \mathrm{dr}$., day 17: $20 \mathrm{dr}$.

$137(=76) \quad$ for marriage contracts, day 16 :

total $40 \mathrm{dr}$.

$20 \mathrm{dr}$.

$138(=77)$

for trierarchêma, [day . :

$60 \mathrm{dr}$.

$139(=78)$

day 22: $3 \mathrm{dr}$.

total $63 \mathrm{dr}$. 
$140(=79) \quad$ for the $6^{\text {th }}$ year for crown tax from the 100 -aroura men, day 18 :

$5 \mathrm{dr}$.

$141(=80) \quad$ for the $2^{\text {nd }}$ year for crown tax from the 100-aroura men, day 18: 5 dr. 5 ob.

142 (= 81) total for "untouched" in bronze at $26.5 \mathrm{ob} .73 \mathrm{dr} .5 \mathrm{ob}$.

143 (=82) agio 7 dr. 4 ob. 1 ch., total 81 dr. 3 ob. 1 ch.

$144(=83) \quad$ for tax on textiles

$145(=84) \quad$ for the $13^{\text {th }}$ year for woollen products

$146(=85)$ for the city day 12

$147(=86)$ for the meris of Polemon day 12

$148(=87)$ for the meris of Themistos day 12

$149(=88)$ for the meris of Herakleides day 12

$304 \mathrm{dr}$.

$150(=89)$ total in bronze at $26.5 \mathrm{ob}$. 297 dr. 1 ob.

$151(=90)$ agio

$152(=91)$

total $1275 \mathrm{dr} .3 \mathrm{ob}$.

254 dr. 4 ob. 299 dr. 2 ob. 1155 dr. 1 ob. 120 dr. 2 ob.

col. vi

\begin{tabular}{|c|c|}
\hline \multirow{2}{*}{\multicolumn{2}{|c|}{$\begin{array}{ll} & \text { one or two lines missing at the top } \\
153(=214+92) & \text { for the } 13^{\text {th }} \text { year [ }\end{array}$}} \\
\hline & \\
\hline $154(=215+93)$ & for bivouacs [ ] ] $2.5 \mathrm{ob}$. , day 15: $23 \mathrm{dr} .2 .5 \mathrm{ob}$. \\
\hline $155(=216+94)$ & day $16: . .$. [] 17 dr., 28 dr. 2 ob., total [. .] \\
\hline $156(=95)$ & [ ] $\quad$ ] 108 dr. 3.5 ob. \\
\hline $157(=217+96)$ & $\ldots[\quad]$. for the hunt of the \\
\hline $158(=218+97)$ & $\begin{array}{l}\text { elephant[s], day 16: in bronze } 4 \text { dr. } 0.5 \text { ob., day 17: in bronze } \\
4 \text { dr. } 0.5 \text { ob. }\end{array}$ \\
\hline $159(=219+98)$ & day 28: in bronze 4 dr. 0.5 ob. [ \\
\hline $160(=99)$ & for horse feed, day 17: [ \\
\hline $161(=100)$ & price of a horse, day 14: $61 \mathrm{dr}$., day 15: [3 dr.], total $64 \mathrm{dr}$. \\
\hline $162(=101)$ & total in bronze at $26.5 \mathrm{ob} .238 \mathrm{dr} .1 .25 \mathrm{ob}$., in bronze .. dr. $2.5 \mathrm{ob}$. \\
\hline $163(=102)$ & for the $12^{\text {th }}$ year for bivouacs \\
\hline $164(=103)$ & $\begin{array}{l}\text { from the professional soldiers, day } 15: 8 \mathrm{dr} .3 .5 \mathrm{ob} ., 4 \mathrm{dr} \text {., total } \\
12 \mathrm{dr} .3 .5 \mathrm{ob} \text {., day } 17: \text {. [ ] . }\end{array}$ \\
\hline $165(=104)$ & day 21: 1 dr. 2.25 ob., total 17 dr. 3.5 ob. in bronze [..] \\
\hline $166-167(=105-$ & -106) two lines illegible \\
\hline $168(=107)$ & . ...... day 5: 1 dr. [ \\
\hline $169(=108)$ & one line illegible \\
\hline $170(=109)$ & for [the .. ${ }^{\text {th }}$ year] for bivouacs [. . dr.] \\
\hline $171(=110)$ & for [the..${ }^{\text {th }}$ year] for crown tax from the professional soldiers . \\
\hline $172(=111)$ & for the $2^{\text {nd }}$ year, for bivouacs from the cleruchs, day 17: $4 \mathrm{dr} .3 \mathrm{ob}$. \\
\hline $173(=112)$ & rse feed, day 17: 5 dr. 1.5 ob., total 9 dr. 4.5 ob. \\
\hline
\end{tabular}


W. Clarysse, D.J. Thompson and L. Capron, An early Ptolemaic bank register

$174(=113) \quad$ total for provisions in bronze at $26.5 \mathrm{ob} . \quad 268 \mathrm{dr} .2 \mathrm{ob}$.

$175(=114) \quad$ agio $28 \mathrm{dr}$.

$176(=115) \quad$ in bronze $\quad 53 \mathrm{dr} .0 .5 \mathrm{ob} .1 \mathrm{ch}$.

$177(=116) \quad$ total in bronze $349 \mathrm{dr} .2 .5 \mathrm{ob} .1 \mathrm{ch}$.

178 (= 117) for the (bank?) through Aristodemos

$179(=118) \quad$ for the $8^{\text {th }}$ year for hides, day 18: $4 \mathrm{dr}$.

$180(=119) \quad$ agio 2.5 ob., total 4 dr. 2.5 ob.

$181(=120) \quad$ for the (account) of the dyke tax of the $13^{\text {th }}$ year

$182(=121) \quad$ for sacred land for the meris of Polemon at Mouchis $3 \mathrm{dr} .2 \mathrm{ob}$.

$183(=122) \quad$ for the meris of Themistos at Arsinoe ...

col. vii

$184(=123) \quad$ for the meris of Herakleides at Philadelph[eia . .

$185(=124) \quad$ total $18 \mathrm{dr} .$.

$186(=125) \quad$ and from the 100-aroura men (?), day 5: $16 \mathrm{dr}$. [4 ob.

187 (= 126) $\quad 16$ dr. 4 ob., total 83 dr. 2 ob. . . [

188 (=127) day 12: 16 dr. 4 ob., day 16: 16 dr. 4 ob., day 18: [16 dr. 4 ob.]

$189(=128) \quad$ illegible

$190(=129) \quad$ [ ] day $25: 16 \mathrm{dr}$. [4 ob.

$191(=130) \quad$ total from the 100-aroura men $466 \mathrm{dr} .4 \mathrm{ob}$.

$192(=131) \quad$ and from the professional soldiers, day 10: $19 \mathrm{dr}$. $4.5 \mathrm{ob}$., day $2[0+$

193 (= 132) 32 dr. 4.5 ob., 19 dr. 5.25 ob., 21 dr. 4.5 ob., 54 dr. 1 ob.

194 (= 133) $\quad .1$ dr. 2 ob., 55 dr. 4.5 ob., 26 dr., 26 dr., total 3[00+ dr.]

195 (=134) illegible

$196(=135) \quad$ agio[

$197(=136) \quad$ in bronze [

$198(=137) \quad$ total in bronze [

$199(=138) \quad$ from the [

$200(=139) \quad$ illegible

$201(=140) \quad$ for the .....

$202(=141) \quad$ day 5: . . dr., day 10: $3 \mathrm{dr}$.

203 (=142) 120 dr., 20 dr., total 26[0+ dr.

$204(=143) \quad$ for the ... [

205 (=144) for Eutychos [

$206(=145) \quad$ day $21: 2$ (tal.) $4[000+\mathrm{dr}$.

$207(=146) \quad$ and for allowances (?) [

$208(=147) \quad$ day $3: 2990 \mathrm{dr} .3 .75 \mathrm{ob}$. 
Verso (in a different hand on the back of column i)

1 from all sources in bronze

279 tal. 3938 dr. 2 ob. 1 ch.

34 tal. 490 dr. 2.25 ob.

$4 \quad 618$ dr. 5.25 ob.

$5 \quad 829$ dr. 4 ob.

$62856 \mathrm{dr} .4 \mathrm{ob} .5 \mathrm{ch}$.

$7 \quad 560 \mathrm{dr}$.

$82002 \mathrm{dr}$.

$9 \quad 14$ tal. 2275 dr. 2.25 ob. 1 ch.

Notes

3 The first four letters of $\Theta \varepsilon \mu$ í $\sigma \tau$ ov are on a small fragment which has been slightly displaced to the left.

4-5 Though the traces are faint, it seems certain that the scribe, either inadvertently or on purpose, repeated the total of 1.4 at the beginning of 1.5 . Apparently he first put down two figures for the whole meris, and then separate figures for the villages (perhaps toparchy centres) of Philadelpheia in the north and Kerkesoucha in the south.

$93 \mathrm{dr} .3 \mathrm{ob}$. must have stood at the start of the line.

15 The end of this line does not contain any figure (the figure that we read baffled us in the $e d$. princ.). It simply repeats $\pi \alpha \rho \grave{\alpha} \mu 1(\sigma \theta 0 \varphi$ ó $\rho \omega v)$ from line 11 . In front of this line there are traces of a line in a previous column, now lost.

16 As often in this text the tax is repeated in abbreviated form at the end of the section $\varepsilon() \varphi v(\lambda \alpha \kappa \iota \tau \iota \kappa о v)$ (1. 21). Although we now read $\mu$ o before $\varphi v \lambda \alpha \kappa \iota \tau($ เкой), the traces preceding this do not fit the expected $\varepsilon \rho \eta-$. As it would also be strange

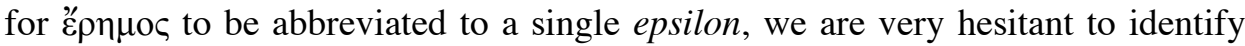
this tax as the erêmophylakitikon, even though no other solution seems possible.

49 For the reading, see the note on $11.79-80$ below.

59 The reading $\dot{\alpha} \pi \alpha \lambda \alpha \gamma \eta \underline{n} \varsigma$ is not assured because the papyrus is damaged and a small correction (substituting a double for a single lambda) is needed. But the word fits the context well: grain taxes are changed into money.

61-62 In 1.61 the figure for Polemon looks very like 234; there is definitely a figure after the lambda. On the right hand fragment we now read Mov́ $\chi \varepsilon(\omega \varsigma)$ followed by a day date and $4 \mathrm{dr}$. Adding this to the $234 \mathrm{dr}$. for Polemon in the same line should give a total of 238 (rather than 234) in 1. 62. The scribe probably made a mistake. 
74 It is just possible to read ( $\gamma^{\prime} v$.) $\zeta v(\tau \eta \rho \hat{\alpha} \varsigma) \pi$ : the tax is abbreviated by a zeta (nearly completely in lacuna) surmounted by a flat ypsilon. Because the figure $\pi$ is divided over two fragments, it appears rather broad.

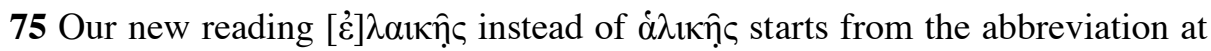
the end of this section, which is clearly $\dot{\varepsilon} \lambda($ ). Lambda and alpha are often very similar in this hand and the start of the lines is indeed lost at this point. For the oil tax, see Cl. Préaux, L'économie royale (1939), pp. 91-92.

The figures cannot be read with certainty, but we should probably read $: \bar{\eta} \kappa \delta \bar{l}$

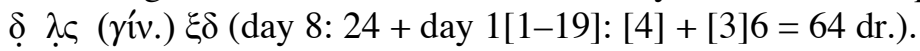

77 Here we have corrected $] \mu \mu \varepsilon v \omega v$ of the ed. princ. into $[\beta] \alpha \lambda \alpha v \varepsilon \varepsilon^{\prime}[\omega] \nu$. Again, as in 1.75 , one letter is lost at the start. The similarity between cursive my, alpha and lambda made the reading difficult, but once recognised it is not in doubt. For the bath tax in the Ptolemaic period, see Préaux, L'économie royale, pp. 338-343.

78 Traces of this line are divided over three fragments and nearly illegible. We expect the name of a second meris or a banking centre such as Arsinoe, but not enough is preserved to allow a reading.

79-80 The abbreviation here stands for $\pi v($ ) or $i \pi($ ) and is different from that of $\mu \mathrm{r}($ ) as found in our text. In the ed. princ. we were misled by the expression $\iota^{\prime} \xi \omega \mu(\sigma \theta \omega \sigma \sigma \varepsilon \omega \varsigma)$, but we now prefer to understand payments "outside the gate" and "inside the gate". At the gate there was certainly a customs house, where the tax of $1 \%$ on transport was paid (for this tax in the Ptolemaic period, see P.Thomas 3) and also the ferry tax (porthmis) for crossing a canal (for this tax, see S. Vinson, The Nile Boatman at Work, Mainz 1998, p. 70). We have not, however, found any parallel passage where "outside" and "inside the gate" are contrasted as here, though the expression $\dot{\varepsilon} \xi \omega \pi \nu \lambda i t n \varsigma$, common in later periods, shows that this kind of localisation was possible. Compare also the $\dot{\varepsilon} \pi \imath \tau \eta \rho \eta \tau \alpha \grave{~} \varepsilon^{\prime} \xi \omega \pi \dot{v}(\lambda \eta \varsigma)$ in

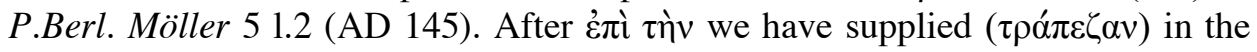
translation, but this is far from certain.

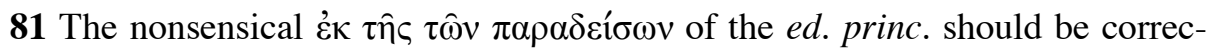

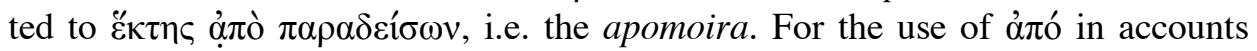
instead of the usual genitive, see, e.g., E. Mayser, Grammatik der griechischen Papyri II.2, p. 378.

84 A small unpublished fragment has been added to the end of this line.

85 Expected is $\overline{i c} \alpha \alpha$ ( $\gamma^{\prime}$ v.) $\beta$ and the minimal traces can be made to fit that reading.

The figure missing at the end of the line should amount to $5 \mathrm{dr}$. if the end figure in 1.86 is read as $25 \mathrm{dr} .3 \mathrm{ob}$., or $9 \mathrm{dr}$. if the end figure is $29 \mathrm{dr} .3 \mathrm{ob}$. The traces visible after the day date 18 do not, however, appear to fit either. Perhaps another day date was given in the lacuna after this.

89 The two signs at the start of the line are heavily damaged, but they cannot contain a figure, since the total of $17 \mathrm{dr} .3 \mathrm{ob}$. corresponds to the sum of $17 \mathrm{dr}$. $1 \mathrm{ob} .+2 \mathrm{ob}$. 
90-109 The top part of col. iv (earlier col. iii) remains unchanged. Former Fragments $\mathrm{C}$ and $\mathrm{F}$ have now been added to the lower part of this column.

90-92 On the pattern of other years, the phylakitikon was probably recorded in these and the missing lines above. The reading 'day 27 ' follows the removal (after photography) of some fibres covering the surface.

105-106 The subtotal of $14 \mathrm{dr} .3 \mathrm{ob}$. is almost certainly made up from twice $7 \mathrm{dr} .1 .5 \mathrm{ob}$. and the total clearly starts with $\xi$, but we are unable to read or make sense of the other figures here.

107 The new figure $84 \mathrm{dr} .1 .5 \mathrm{ob}$., which is the result of the join between former Frags. A and B, is the sum of $7 \mathrm{dr} .+77 \mathrm{dr}$. $1.5 \mathrm{ob}$. in 11. 99-104. The figure of 1. 106, lost in the lacuna, is not included; this apparently represents a payment made in chalkos isonomos, which is calculated separately in 1. 108.

109 There is a descending stroke which could be the rho of 100 in $\dot{\varepsilon} \kappa \alpha \tau o v \tau \alpha-$ poú $\omega \mathrm{v}$; the following sign is not incompatible with the abbreviation for aroura.

115-116 A small unpublished fragment has been inserted here to the left of the nearly illegible old 11. 227-228.

128-133 Composite totals are here recorded for arrears from years 7-12, which have been detailed in 11. 71-127.

135 Only a few horizontal fibres are preserved. The traces at the end of the word do not allow the reading ópoגoy!

153 Here we have been able to insert former Frag. D. The join is confirmed by the continuation of the fibres and because the phi in i $\pi \pi$ ov $\tau \rho о \varphi \eta \hat{~}$ (1. 160) is divided over the main fragment (at the foot) and Frag. D.

157 The possibility of reading $\theta \underline{\text { ṇ }}$ $\alpha \varsigma$ is suggested by the parallel text $S B$ XII 1077111.5 and 16. The traces are faint, however, and do not fit the reading well.

158 Payments of 4 dr. 0.5 ob. preceded by $\chi \alpha(\lambda \kappa o v)$ clearly include the agio at the rate of $24.5 \mathrm{ob}$. to a tetradrachm, cf. 1. 67.

176 The translation of the ed. princ. was wrong. We have corrected $53.5 \mathrm{dr}$. 1 chalkous into 53 dr. 0.5 ob. 1 chalkous.

191 The total at the end of the line is not easy to make out but the reading is not in doubt; this represents the dyke tax payment for twenty-eight 100-aroura men at $1 \mathrm{ob}$. an aroura. 